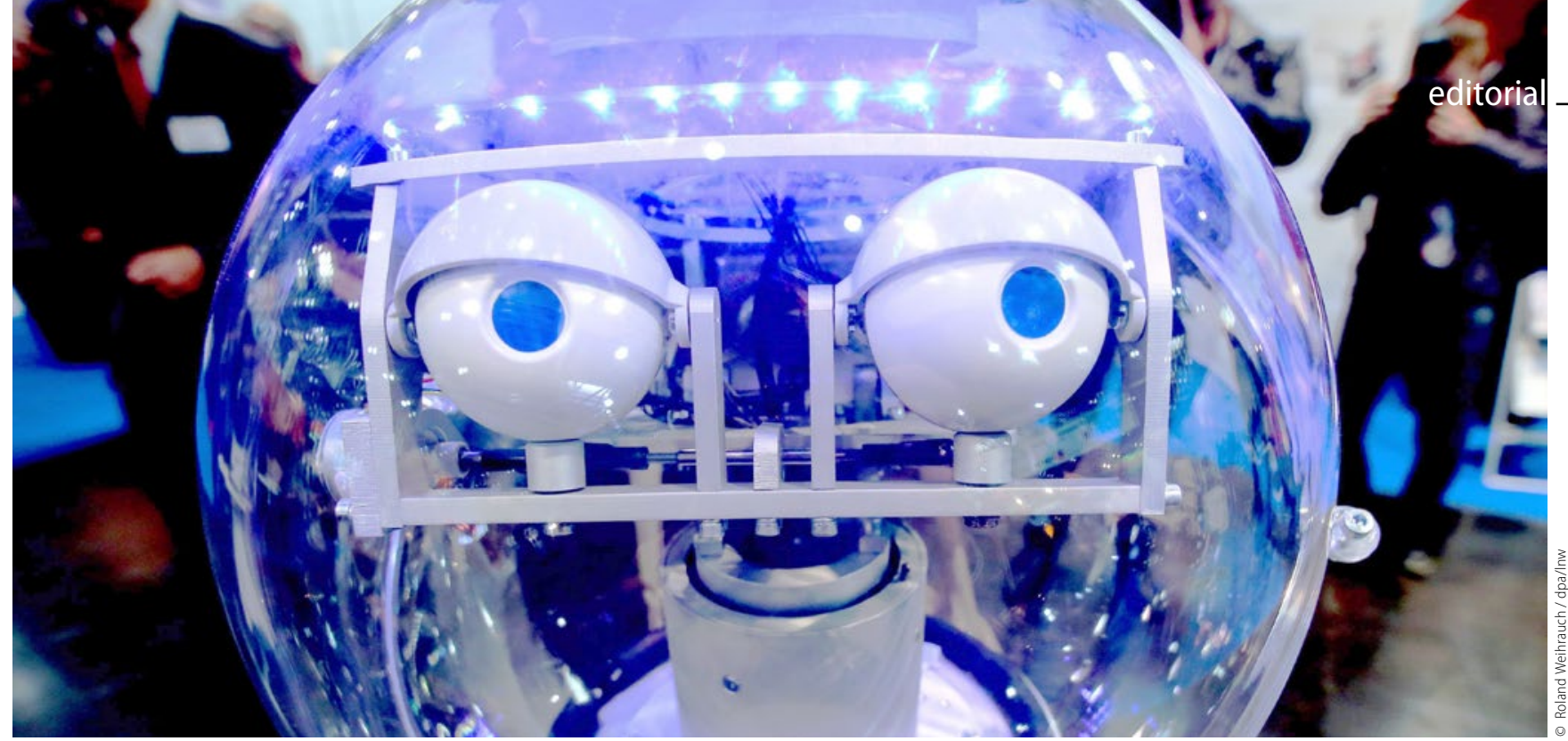

Technische Errungenschaften erleichtern den Alltag, auch in der Diabetologie. Aber sie sollten nicht allein stehen.

\title{
Wie viel Technik verträgt die Diabetologie?
}

Der Kardiologe und Friedensnobelpreisträger Bernard Lown schreibt in seinem großartigen Werk [1]:,Die verlorene Kunst des Heilens“: „In unserem modernen Zeitalter nährt die zunehmende Nähe von Medizin und Wissenschaft die Illusion, beide seien identisch. Sie verführt die Ärzte dazu, die Bedeutung des Einsatzes am Krankenbett zu verharmlosen, begünstigt die Vernachlässigung einer umfassenden Anamnese und vermindert das eigene Bemühen, menschliche Beziehungen zu den Patienten aufzubauen.

Der Fokus verschiebt sich vom Heilen zum Behandeln, so, als ob diese beiden Elemente eher gegensätzliche als komplementäre Systeme wären. Bei den Patienten verstärkt die wissenschaftliche Revolution die Erwartung, dass jede wie auch immer geartete Krankheit sofort behandelt werden könne.“ Lown beschreibt einen Prozess, der augenblicklich in der Diabetologie zu beobachten ist, getriggert durch die Einführung einer neuen Technologie: die Glukosesensoren.

\section{Ein neues Gewand für die Diabetestherapie}

Medizin und Technik - in vielen Bereichen des medizinischen Alltags sind diese beiden feste Partner. Und wir können uns kaum mehr vorstellen, wie es ohne die technischen Errungenschaften möglich war, Patienten zu behandeln. Denken wir nur an Herzschrittmacher, Dialysegeräte, Herz-Lungen-Maschine, Gelenkprothesen oder, im Falle der Diabetologie, Blutzuckermessgeräte, Insulinpens und Insulinpumpen. Nun betritt ein neuer "Player" das Feld - die Glukosesensoren erleben einen wahrhaften Boom, seitdem der Gesetzgeber die rtCGM-(real time continous glucose measurement) Systeme als Hilfsmittel eingestuft hat.

In der Diabetologie bedeutete Technik bislang die Weiterentwicklung von Insulinspritzen und Blutzuckermessgeräten. Ich kann mich noch gut daran erinnern, wie zu meiner Assistenzarztzeit genau zwei Blutzuckermessgeräte auf dem Markt waren. Heute kaum mehr vorstellbar: Eine Blutzuckerbestimmung dauerte 1 bis 2 Minuten, erforderte einen Blutstropfen von etwa
$20 \mu \mathrm{l}$ und kostete etwa 500 Deutsche Mark! Inzwischen sind mehr als 60 verschiedene Geräte auf dem Markt- , zu kaufen gibt es sie im Discounter, für eine Messung benötigt man etwa $3 \mu \mathrm{l}$ Blut und sie dauert nur wenige Sekunden. Während sich die verfügbaren Blutzuckermessgeräte und Insulinpumpensysteme in den vergangenen 20 Jahren rapide verbessert haben, gab es im Bereich der Sensortechnologie aber über viele Jahre keine wesentliche Entwicklung im Hinblick auf eine praktikablere Alltagslösung.

Nun haben wir sogar den Eindruck, dass der Traum von einem Closed-loop-System demnächst wahr werden könnte. Die Koppelung zwischen Insulinzufuhr und dem aktuell gemessenen Blutzucker durch einen Glukosesensor, basierend auf individuellen Algorithmen, könnte die Diabetestherapie revolutionieren.

\section{Zur guten Therapie gehört mehr als ein Sensor}

Dreh- und Angelpunkt eines sinnvollen Einsatzes von rtCGMSystemen scheint mir die Schulung des Patienten im Umgang mit der neuen Technologie zu sein. Denn wer sein Handwerk nicht beherrscht und Trendpfeile nicht zu interpretieren weiß oder aber bei Defekt des Sensors keinen Plan B hat, ist in Gefahr. Mit dem Schulungsprogramm Spectrum haben wir die

\section{Fortsetzung auf Seite 4}

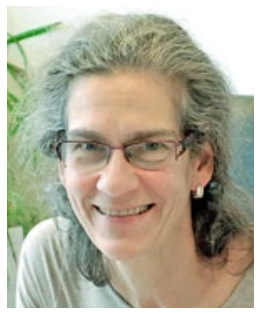

Dr. med. Veronika Hollenrieder

FÄ für Innere Medizin, Diabetologin DDG Hauptstr. 14, 82008 Unterhaching veronika@praxis-hollenrieder.de 
Möglichkeit, bereits im Vorfeld (Unterrichtseinheit 1) zu klären, welche Erwartungen ein Patient hat und inwieweit sich diese durch den Einsatz eines Glukosesensors realisieren lassen.

Auch ist vor jeder Verordnung zu klären, inwieweit der Patient die Grundregeln der ICT-Therapie mit BE-und Korrekturfaktoren, Insulinwirkung sowie die BE-Berechnung beherrscht. Das ist durch eine ausführliche Protokollierung überprüfbar. In Ausnahmefällen (Kinder, psychiatrische Patienten, Schwangerschaft) kann dies unter Umständen auch durch pflegerisch tätige Personen erfolgen. Da der Gesetzgeber keine klaren Richtlinien zur Dokumentation vor Antragstellung festgelegt hat, erleben wir augenblicklich bei der Genehmigung durch Krankenkassen oder den medizinischen Dienst viel Divergenz ( $\triangleright$ s. Beitrag Info Diabetologie 3-17, S. 47; Glukosesensor auf Kasse: Wer zahlt am Ende drauf?; online: www.springermedizin. de/info-diabetologie). Seit 8/2017 gibt es nun aber einen Konsens zwischen FKDB und MDK ( $\diamond$ s. Kasten)

Inzwischen haben wir in unseren Diabeteszentren auch so manche Erfahrung im Umgang mit den verfügbaren Systemen gemacht. Wie so häufig in der Medizin unterscheiden sich die Erwartungen von Patienten und Ärzten deutlich. Während es uns Therapeuten vordergründig um die Verbesserung der Blutzuckereinstellung geht, sind Patienten vor allem an einem Plus an Lebensqualität interessiert. Weniger zu messen, diskret über den aktuellen Blutzucker Bescheid zu wissen und bei Über-oder Unterschreiten von Alarmgrenzen benachrichtigt zu werden übt definitiv eine große initiale Faszination aus. Es ist allerdings nur die Basis für eine korrekte Insulintherapie. Dabei müssen Bewegung, Sport, Alkohol, Infekte um nur einige zu nennen, natürlich wie bislang auch in das Handeln einfließen.

\section{Psychologie nicht vergessen}

Nicht zu vernachlässigen ist meines Erachtens auch der psychologische Effekt eines Glukosesensors. Mit der permanenten Visualisierung der Blutzuckerwerte muss man erst umgehen lernen. Da gibt es den Patiententypus, der seine eigene Wahrnehmung über das System trainieren lernt und Blutzuckerschwan-

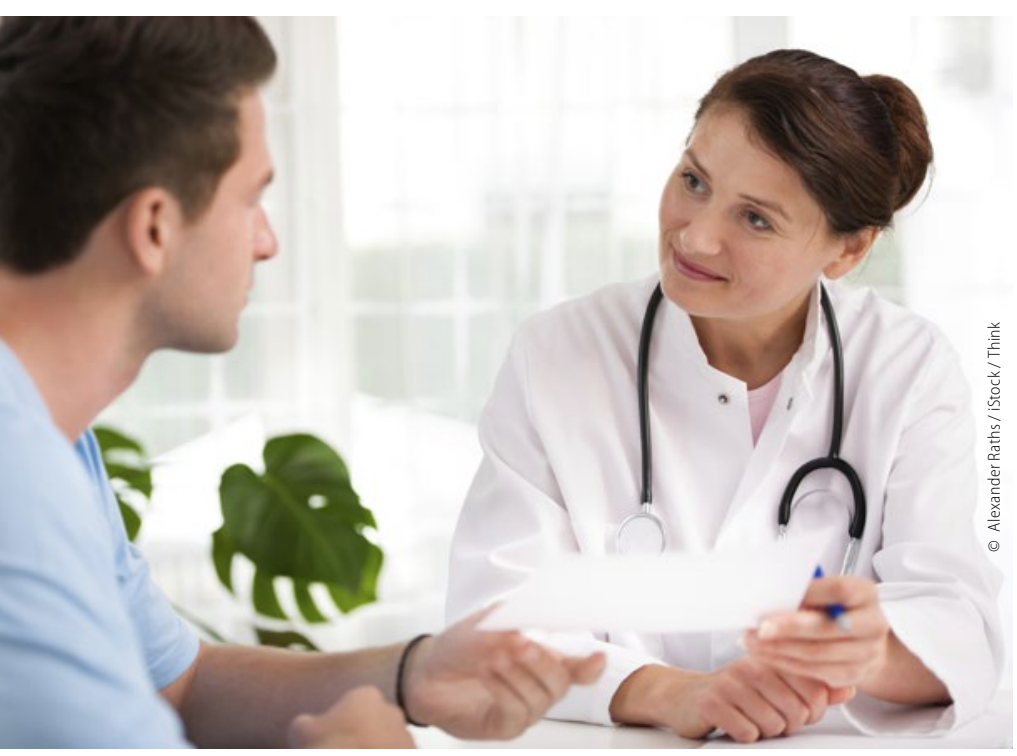

Das empathische Gespräch zwischen Arzt und Patient kann auch eine hochentwickelte Technik nicht ersetzen.

\section{Kostenerstattung kontinuierliche Glukosemessung}

\section{Welche Voraussetzungen sollten erfüllt sein?}

Die Fachkommission Diabetes in Bayern e.V. (FKDB) hat einen Konsensus mit den Medizinischen Dienst der Krankenkassen (MDK) Bayern zur Indikationsstellung für Kontinuierliche Interstitielle Glukosemessung mit Real-Time-Messgeräten (rtCGM) erstellt. Neben den Anforderungen, die der Gemeinsame Bundesausschuss an die Indikationsstellung hat, wird etwa darüber informiert, welche Unterlagen seitens des Antragsstellers (Patient) zur Begutachtung durch den MDK benötigt werden und wie diese Unterlagen zusammenzustellen sind. Zudem wird erläutert, wann von einem therapeutischen Nutzen durch rtCGM ausgegangen werden kann und bei welchen Patienengruppen besondere Bedingungen vorliegen.

Das Fazit der Beteiligten: Durch Objektivierung von praxisrelevanten und realistischen Kriterien zur Antragsstellung eines rtCGM-Gerätes können Genehmigungsverfahren für alle Beteiligten vereinfacht und transparent werden. Klare Vorgaben für die Patienten sind erforderlich, um einen therapeutischen Nutzen dokumentieren zu können.

\section{- Das Konsensuspapier ist über die FKDB aus dem Internet zu laden: http://www.fkdb.net/wp-content/uploads/2017/09/ Konsensus-zwischen-MDK-und-FKDB_08-2017-2.pdf}

Quelle: Fachkommission Diabetes in Bayern (FKDB)

kungen verringert. Aber eben auch den, der seine schlechten Werte mental ausblendet oder sich von den häufigen Alarmen gestört fühlt und sie ignoriert oder gar ausschaltet. Und es gibt die „Hypo-Surfer“ - die in dem Glauben an die Technik allen ärztlichen Ratschlägen zum Trotz ihre Zielwerte weiter absenken und dadurch ihre Hypoglykämie-Wahrnehmungsstörung verstärken. Eine Entlastung verspüren definitiv die Lebenspartner der Betroffenen, die vor allem Hypoglykämien beim Autofahren, Sport oder während der Nacht fürchten. Aber auch Sie müssen wissen, dass eine größere Sicherheit nur gegeben ist, wenn das System funktioniert und ordnungsgemäß benutzt wird.

Eine neue Technologie ist immer so gut wie ihr Anwender, auch sind technische Defekte oder Hautirritationen bis hin zu Kontaktallergien möglich. Es genügt also nicht, moderne Technologien anzuwenden, sondern wir Ärzte müssen sie in unser therapeutisches Gespräch integrieren. Ein stimmiges Arzt-Patienten-Verhältnis ist somit durch die Technologie nicht überflüssig geworden sondern gewährleistet deren therapeutischen Nutzen. Deshalb sollte eine sprechende Medizin auch adäquat honoriert werden, was bislang leider nicht der Fall ist.

Zweifelsohne haben wir mit den Glukosesensoren nun ein Werkzeug in der Hand, das enorme diagnostische und therapeutische Möglichkeiten eröffnet. Aber wir alle, die wir diese Systeme nutzen, müssen lernen, damit sorgsam umzugehen und Erwartungen nicht zu hoch zu schrauben. Dann können sie unsere therapeutischen Bemühungen unterstützen, nicht aber das Gespräch mit Patienten ersetzen. Dafür werden auch künftig Vertrauen, Empathie und diabetologische Expertise erforderlich sein.

Literatur

1. Berard Lown: Die verlorene Kunst des Heilens, Anleitung zum Umdenken. Suhrkamp 14.Aufl. 2017, suhrkamp taschenbuch 3574 (Originalausgabe 1996 "The Lost Art of Healing") 